\title{
MicroRNA-29 overexpression by adeno- associated virus suppresses fibrosis and restores muscle function in combination with micro-dystrophin
}

\author{
Kristin N. Heller, ${ }^{1,2}$ Joshua T. Mendell, ${ }^{3,4}$ Jerry R. Mendell, ${ }^{1,2}$ and Louise R. Rodino-Klapac ${ }^{1,2}$ \\ 'Center for Gene Therapy, The Research Institute at Nationwide Children's Hospital, Columbus, Ohio, USA. \\ 2Department of Pediatrics and Neurology, The Ohio State University, Columbus, Ohio, USA. ${ }^{3}$ Department of Molecular \\ Biology, ${ }^{4}$ Howard Hughes Medical Institute, University of Texas Southwestern Medical Center, Dallas, Texas, USA.
}

\begin{abstract}
Duchenne muscular dystrophy (DMD) is caused by dystrophin deficiency resulting in progressive muscle weakness and fibrotic scarring. Muscle fibrosis impairs blood flow, hampering muscle repair and regeneration. Irrespective of the success of gene restoration, functional improvement is limited without reducing fibrosis. The levels of miR-29c, a known regulator of collagen, are reduced in DMD. Our goal is to develop translational, antifibrotic therapy by overexpressing miR-29c. We injected the gastrocnemius muscle with either self-complementary AAV.CMV.miR-29c or singlestranded AAV.MCK.micro-dystrophin alone or in combination in the $m d x / \mathrm{utrn}^{+/-}$mouse, a DMD mouse model. Treatment of 3-month-old $m d x / \mathrm{utrn}^{+/-}$mice with AAV.miR-29c showed a reduction in collagen and increased absolute and specific force compared with untreated animals, but neither parameter reached WT levels. Combinatorial gene delivery in 3-month-old $m d x / \mathrm{utrn}^{+/-}$mice further decreased fibrosis, and showed a reduction of transcript levels for Col1A, Col3A, fibronectin, and Tgfb1. In addition, absolute and specific force was normalized and equivalent to WT. However, protection against eccentric contraction fell short of WT levels at this time point. When this same mouse model was treated with miR-29c/micro-dystrophin combinatorial therapy at 1 month of age, there was complete normalization of specific and absolute force and protection against eccentric contraction-induced injury was comparable to WT. These findings highlight the potential for miR$29 \mathrm{c}$ as an important addition to the armamentarium for translational gene therapy, especially when used in combination with micro-dystrophin in DMD.
\end{abstract}

Conflict of interest: K.N. Heller, J.R. Mendell, and L.R. Rodino-Klapac are inventors on patent application number 62/323,163 for the use of miR-29 in combination with micro-dystrophin. L.R. Rodino-Klapac has received a license option distribution fee from Sarepta Therapeutics, Inc. J.R. Mendell has received consulting fees from Sarepta Therapeutics, Inc. and Avexis, Inc. J.T. Mendell has received consulting fees from RNA Newco, Inc.

Submitted: February 10, 2017

Accepted: April 4, 2017

Published: May 4, 2017

\section{Reference information:}

JCI Insight. 2017;2(9):e93309. https:// doi.org/10.1172/jici.insight.93309.

\section{Introduction}

Duchenne muscular dystrophy (DMD) is the most common, severe childhood form of muscular dystrophy and is caused by dystrophin deficiency resulting in muscle loss, progressive weakness, and fibrotic scarring. Current treatment strategies focus on replacing the missing dystrophin gene. We and others have shown that micro-dystrophin treatment increases tetanic force and protects from eccentric contraction-induced damage in the $m d x$ mouse, which does not express dystrophin (1-4). A caveat to gene replacement alone is that it fails to address fibrosis and fat replacement resulting from muscle fiber loss. In contrast, the miR-29 family, comprised of miR-29a, b, and c, is implicated in fibrosis in a variety of organs including liver, lung, heart, kidney, and skeletal muscle, and provides a potential tool for translational therapy (5-11). miRs are noncoding, single-stranded RNAs, of 21-25 nucleotides in length that constitute a novel class of gene regulators. They negatively regulate their targets at the post-transcriptional level by binding to the 3 ' untranslated regions (UTRs) of miRs, triggering accelerated mRNA decay and reduced translation. miR-29 family members are generally reduced in fibrotic states and, accordingly, the potential for delivery of these miRs to downregulate collagen and other constituents of fibrosis has gained attention as a potential antifibrotic therapeutic approach (12-15). Delivered as double-stranded oligonucleotides (oligos) or packaged in recombinant adeno-associated virus (rAAV), miR mimics have been developed to normalize gene expression in disease states. In the context of pulmonary fibrosis, gene transfer of miR-29b into an induced pulmonary fibrosis mouse model significantly downregulated Col1A1, Col3A1, and fibronectin mRNA, confirmed by immunohistochemistry 
A
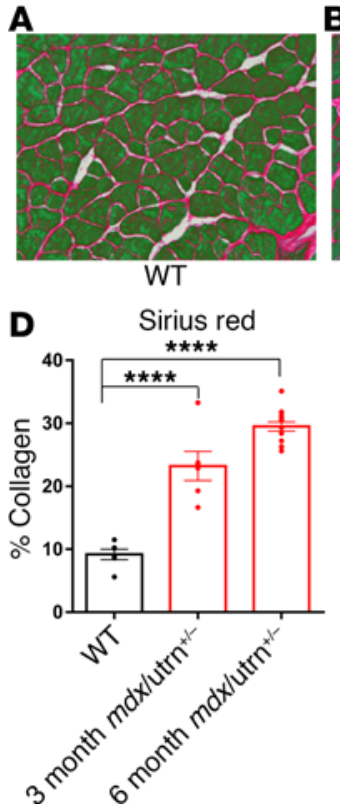

B

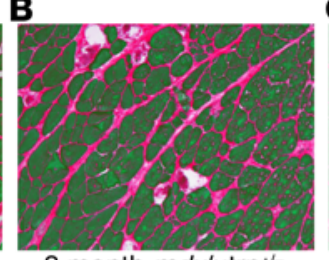

3 month $m d x /$ utrn ${ }^{+/-}$

E

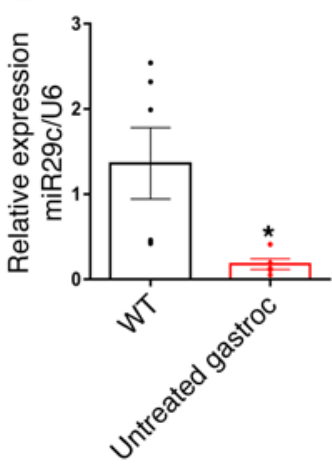

C

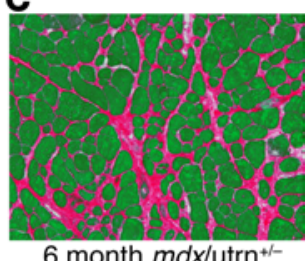

6 month $m d x /$ utrn ${ }^{+/-}$

Figure 1. Fibrosis and miR-29c downregulation in muscle of $m d x$ / utrn $^{+/-}$mice. Picrosirius red staining in WT (A), 3-month-old (B), and 6-month-old (C) $m d x /$ utrn $^{+/-}$gastrocnemius (GAS) muscle. Original magnification, $\times 20$. (D) Quantification of the picrosirius red staining shows a significant increase in collagen at both time points compared with WT muscle ( $n=6$ per group except $n=14$ for 6 -monthold mice); 1-way ANOVA. (E) qRT-PCR analysis shows a significant decrease in miR-29c levels in 6-month-old $m d x /$ utrn $^{+/-}$CAS muscle compared with WT ( $n=5-6$ per group); unpaired 2-tailed Student's $t$ test. All data represent mean $\pm \mathrm{SEM} .{ }^{*} P<0.05,{ }^{* * *} P<0.0001$.

and Western blot (15). Currently, a miR-29b mimic produced by MiRagen Therapeutics is in a phase 1 clinical trial to evaluate safety, tolerability, and pharmacokinetics in healthy volunteers (MRG-201;NCT02603224). As proof of principle, Wang and colleagues showed that delivery of miR-29 mimics to dystrophic muscle resulted in a reduction of Col1A1 and Col3A1 (9). In translating miR-29 delivery to the clinic for DMD, viral delivery of miR-29 using rAAV holds a distinct advantage in that it allows for one-time treatment rather than repeated intravenous delivery of oligos.

In addition to its direct effect on constituents of extracellular matrix (ECM), miR-29 also promotes muscle regeneration via downregulation of Yin Yang 1 (YY1), a transcriptional repressor of myogenesis (16). miR-29 has been also been shown to repress proliferation and promote myoblast differentiation in $\mathrm{C} 2 \mathrm{C} 12$ cells (17). In an experiment to promote regeneration, miR-29c-mimic oligos were injected into the tibialis anterior (TA) muscles of 4 -week-old $m d x$ mice at the peak of muscle degeneration. Treatment was done 3 times per week for 2 consecutive weeks, with muscle collected 3 weeks after the initial injection. Staining for embryonic myosin heavy chain (E-MyHC) revealed an increase in the number of regenerating fibers validated by detection of an increase in myogenin, myoD, and Pax 7 by immunoblotting (9).

An important part of the work to be presented in this report is the study of antifibrotic therapies in the haploinsufficient, $m d x / \mathrm{utrn}^{+/-}$mice $(18,19)$. In addition to a complete absence of dystrophin, this model lacks one copy of utrophin (utrn), which is known to partially compensate for dystrophin loss (20). Gutpell and colleagues showed that as early as 8 weeks of age, the gastrocnemius (GAS) muscle of $m d x /$ utrn $^{+/-}$mice show significantly more fibrosis than $m d x$ mice (19). In the current study, we validated these findings in GAS and found that fibrosis was accompanied by a reduction of miR-29c transcripts. In addition, we provide further evidence that miR-29c/micro-dystrophin combination therapy reduces expression of collagens I and III and Fbn, direct targets of miR-29c $(12,15)$, and $T g f b 1$, which is a negative regulator of miR-29c (9). Treatment lowers overall fibrosis, improves absolute and specific force, and protects against contraction-induced injury. These findings illustrate the therapeutic potential of miR-29c therapies.

\section{Results}

Increased fibrosis and reduced miR-29c levels in $m d x / \mathrm{utrn}^{+/-}$mice. An increase in GAS muscle fibrosis in 3- and 6-month-old $m d x /$ utrn $^{+/-}$mice is clearly demonstrated by picrosirius red staining for collagen I (Col1A) and III (Col3A). Quantified comparisons using picrosirius staining to quantify the percentage of collagen within the total area of the muscle section reveal $9.2 \% \pm 0.8 \%$ in WT versus $23.2 \% \pm 2.3 \%$ in $m d x /$ utrn $^{+/}$by age 3 months, with further increase by age 6 months, reaching $29.5 \% \pm 0.7 \%(P<0.001)$ (Figure 1, A-D). To establish whether increased fibrosis in $m d x /$ utrn $^{+/}$muscle correlated with a reduction in miR-29c, total RNA was extracted from the GAS muscle and quantified by reverse transcriptionPCR (qRT-PCR) for miR-29c expression. miR-29c transcript levels were dramatically downregulated in 6-month-old $m d x /$ utrn $^{+/-}$mice compared with WT (Figure 1E).

Validation of miR-29c activity in vitro and in vivo. AAV vectors have a well-established safety profile in clinical trials and proof of principle has been established for the use of self-complementary AAV (scAAV) for packaging and delivery of miRs in vivo $(21,22)$. Originally, the scAAV vector was made with the natural pre-miR-29c sequence surrounded by $\sim 200$ nucleotides of its endogenous genomic sequence. However, following our initial studies (data not shown) and a report by Knabel et al. (14), which documented limited 
A

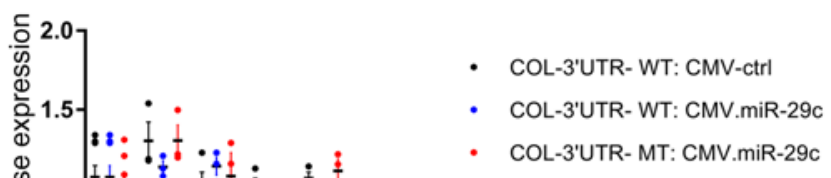

- COL-3'UTR-MT: CMV.miR-29c

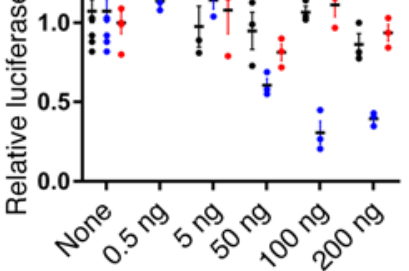

B
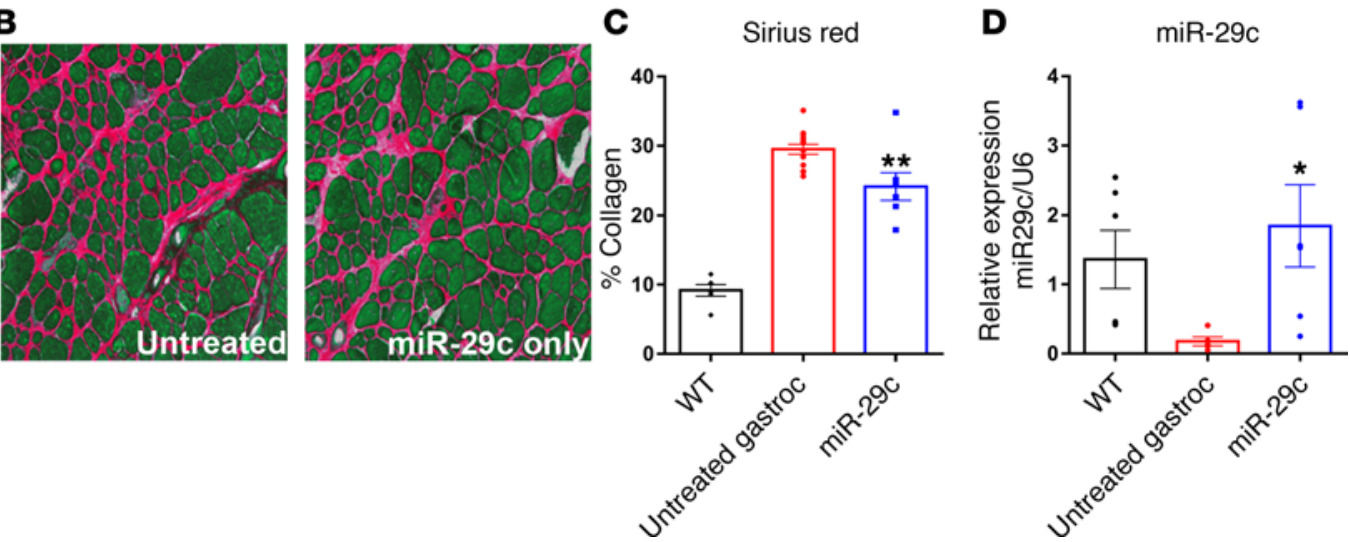

Figure 2. Validation of miR-29c activity in vitro and in vivo. (A) In vitro luciferase assay to test the effect of the scAAV.CMV.miR-29c on one of the downstream targets of miR-29c, COL1A1. (B) Picrosirius red staining revealed a significant decrease in collagen in aav.miR-29c-treated $m d x /{ }^{2}$ trn ${ }^{+/-}$muscle 12 weeks after injection compared with untreated muscle. Original magnification, $\times 20$. (C) Quantification of the picrosirius staining reveals an $18.3 \%$ reduction in collagen compared with untreated muscle ( $n=5-6$ per group); 1 -way ANOVA. (D) qRT-PCR confirms that there is an increase in miR-29c transcript levels in treated muscle ( $n=6-7$ per group except $n=14$ untreated); 1 -way ANOVA. All data represent mean \pm SEM. ${ }^{*} P<0.05,{ }^{* *} P<0.01$.

overexpression of miR-29a when expressed in a similar manner, we constructed an AAV vector containing miR-29c embedded in a miR-30 backbone that is frequently used to express short-hairpin RNAs (23), embedded in an elongation factor $1 \alpha(\mathrm{EF} 1 \alpha)$ intron (24) driven by a CMV promoter. To test the effectiveness of the vector in vitro, a fragment of the COL1A1 3'UTR containing miR-29 binding sites was fused downstream of the firefly luciferase (Luc) gene (14). Cotransfections of the miR-29c plasmid caused substantial repression of luciferase activity and this effect was reversed by the presence of mutations in the miR-29 binding sites (Figure 2A).

For in vivo delivery studies, we performed unilateral i.m. injections $\left(5 \times 10^{11}\right.$ vector genomes $\left.[\mathrm{vg}]\right)$ in GAS in 3-month-old $m d x /$ utrn $^{+/-}$mice. Muscle was harvested 12 weeks after injection and analyzed for fibrosis and miR-29c and ECM component transcript levels. Picrosirius red staining revealed a significant decrease in collagen staining throughout the GAS muscles (Figure 2, B and C) compared with the untreated contralateral $m d x / \mathrm{utrn}^{+/}$GAS muscle. Quantification of the picrosirius red staining using ImageJ software demonstrated that treated muscle had an $18.3 \%$ reduction in collagen compared with the untreated muscle (treated: $23.3 \% \pm 1.3 \%$ vs. untreated: $29.5 \% \pm 0.7 \%)(P<0.05)$ (Figure $2 \mathrm{C}$ ). To confirm overexpression of miR-29c in treated muscle, total RNA was extracted from the GAS muscle for qRT-PCR from 6-month-old $m d x / \mathrm{utrn}^{+/-}$and WT mice. miR-29c was significantly increased in the treated group compared with untreated mice (Figure 2D). These experiments demonstrated that scAAV.CMV.miR-29c increases miR-29c levels and reduces collagen assessed both in vitro and as an in vivo therapy.

scAAV.miR-29c improves muscle force. Replacement of intact muscle with significant accumulation of fibrosis can affect muscle function $(25,26)$. GAS muscle from $m d x / \mathrm{utrn}^{+/-}$mice treated with scAAVrh.74. CMV.miR-29c following i.m. injection of $5 \times 10^{11} \mathrm{vg}$ was assessed 12 weeks after injection. Absolute force was increased compared with untreated $m d x / \mathrm{utrn}^{+/-}$GAS muscle (treated: $2,277 \pm 161.7 \mathrm{mN}$ vs. $m d x /$ utrn $^{+/-}$untreated: $\left.1,722 \pm 145.7 \mathrm{mN}\right)(P<0.05)$ (Figure 3A). Specific force also improved compared with untreated GAS muscle (treated: $204.7 \pm 11.7 \mathrm{mN} / \mathrm{mm}^{2}$ vs. $m d x / \mathrm{utrn}^{+/-}$untreated: $151.6 \pm 14.5 \mathrm{mN} /$ 
A
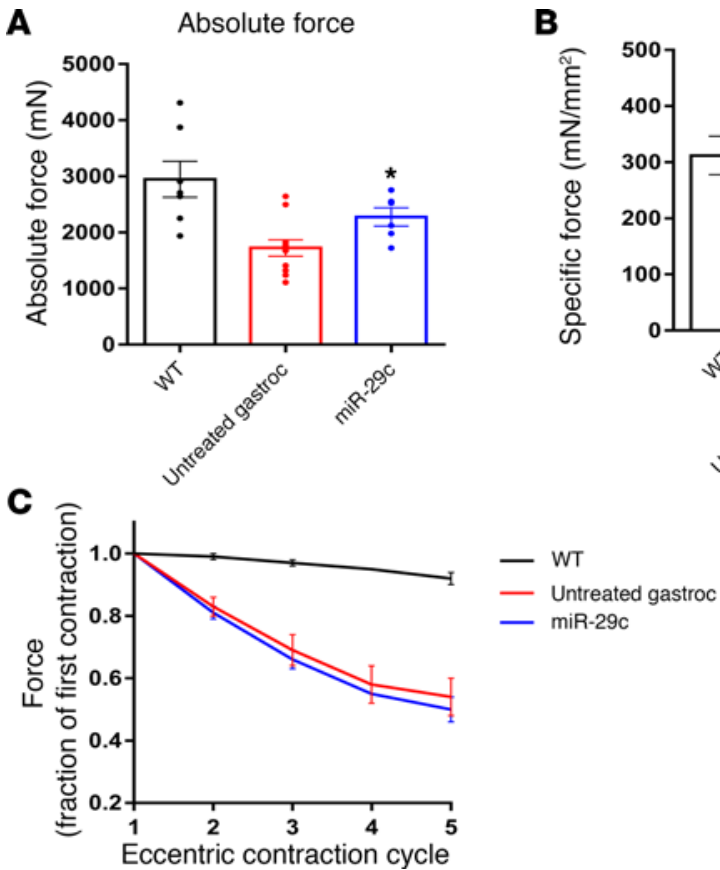

B

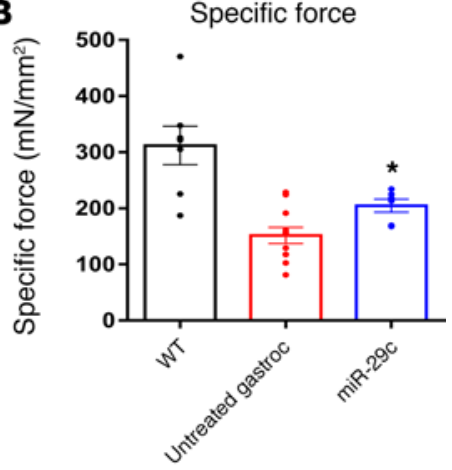

Figure 3. AAV.miR-29c improves muscle force but does not protect against contraction-induced damage. Measurement of absolute (A) and normalized specific force (B) following tetanic contraction in AAV.miR-29c-injected gastrocnemius muscles was significantly increased compared with untreated $m d x /$ utrn $^{+/-}$muscle. $n=6-7$ per group except $n=11$ untreated. ${ }^{*} P<0.05$ by 1 -way ANOVA. (C) Muscles were then assessed for loss of force following repetitive eccentric contractions. Treated mice decayed to the same degree as untreated $m d x / \mathrm{utrn}^{+/-}$ muscles (red). $n=5-6$ per group except $n=9$ untreated; 2-way ANOVA. All data represent mean $\pm \mathrm{SEM}$.

$\left.\mathrm{mm}^{2} ; P<0.05\right)$ (Figure 3B), but failed to reach WT force levels $\left(312.0 \pm 34.1 \mathrm{mN} / \mathrm{mm}^{2}\right)$. Notably, scAAVrh.74. CMV.miR-29c treatment did not improve force decay following repeated eccentric contraction (Figure 3C).

Combinatorial miR-29c/micro-dystrophin therapy is more effective than either alone. To determine whether miR-29c/ micro-dystrophin combinatorial gene delivery would be more beneficial at reducing fibrosis, 3-month-old $m d x /$ utrn $^{+/-}$mice received an i.m. injection to the left GAS muscle with either scAAVrh.74.CMV.miR-29c alone, ssAAVrh74.MCK.micro-dystrophin + scAAVrh.74.CMV.

miR-29c, or ssAAVrh74.MCK.micro-dystrophin alone at $5 \times 10^{11} \mathrm{vg}$. The mice were analyzed at 12 and 24 weeks after injection. Micro-dystrophin expression transduction efficiency in cohorts receiving either combinatorial therapy or rAAV.micro-dystrophin alone both showed comparable numbers of positive muscle fibers $(>70 \%)$ at 12 and 24 weeks (Supplemental Figure 1, A-C; supplemental material available online with this article; https://doi.org/10.1172/jci.insight.93309DS1). The amount of fibrosis was analyzed by picrosirius red staining, which revealed a significant decrease in collagen staining throughout the GAS muscles in all treated groups (Figure $4, \mathrm{~A}$ and B) compared with the untreated contralateral $m d x / \mathrm{utrn}^{+/-}$ GAS muscle. Quantification of the picrosirius red staining showed that muscle cotreated with micro-dystrophin/miR-29c had a $40.8 \%$ reduction in collagen compared with the untreated muscle (combinatorial treatment: $17.47 \% \pm 0.75 \%$ vs. untreated: $29.5 \% \pm 0.7 \%)(P<0.0001)$ (Figure $4 \mathrm{~B})$. To confirm expression of miR-29c, qRT-PCR was performed on the GAS muscle and all treatment groups had an increase in miR29c compared with untreated muscle (Figure 4C).

At 24 weeks after injection, we saw similar results compared with 12 weeks after injection. There was a $47 \%$ reduction in collagen by picrosirius red staining compared with the untreated muscle (combinatorial treatment: $16.5 \% \pm 1.23 \%$ vs. untreated: $31.7 \% \pm 0.92 \% ; P<0.0001)$ and a coincident increase in miR-29c transcript level (Supplemental Figure 2, A-C).

Reduction of ECM expression following combinatorial therapy. To further validate reduction of collagen observed by picrosirius red staining, qRT-PCR was performed on the muscle to quantify transcript levels of $\operatorname{Col1A}, \operatorname{Col} 3 A$, as well as another ECM component, fibronectin $(F b n)$. qRT-PCR analysis detected a decrease in Col1 $A$ and $\operatorname{Col} 3 A$ following each treatment; however, only the cohort treated with both microdystrophin and miR-29c showed significant reduction (Figure 4, D and E). Our analysis revealed that $F b n$ was significantly reduced only in the cotreated cohort (Figure $4 \mathrm{~F}$ ).

TGF- $\beta 1$ has been previously shown to be upregulated in dystrophic muscle, likely playing a role in the initiation of the fibrotic cascade (27-29). TGF- $\beta 1$ is a known profibrotic cytokine that downregulates miR-29c and is responsible for conversion of myoblasts to myofibroblasts, with an increase in collagen and muscle fibrogenesis (9). qRT-PCR analysis shows that cotreated muscle had significantly lower levels of $\mathrm{Tgfb} 1$ compared with untreated muscle and either treatment alone (Figure 4G).

At 6 months after injection, cotreated muscle continued to show reduced $\operatorname{Col} 1 \mathrm{~A}, \operatorname{Col} 3 \mathrm{~A}, \mathrm{Fbn}$, and $\mathrm{Tg} f \mathrm{~b} 1$ levels, whereas only slight reductions in CollA mRNA levels in the miR-29-only and micro-dystrophinonly groups were observed (Supplemental Figure 2, D-G).

Combinatorial therapy restores force and protects from contraction-induced damage. Knowing that miR-29-treated muscle had a modest but significant increase in absolute and specific force, we asked whether combining 
A
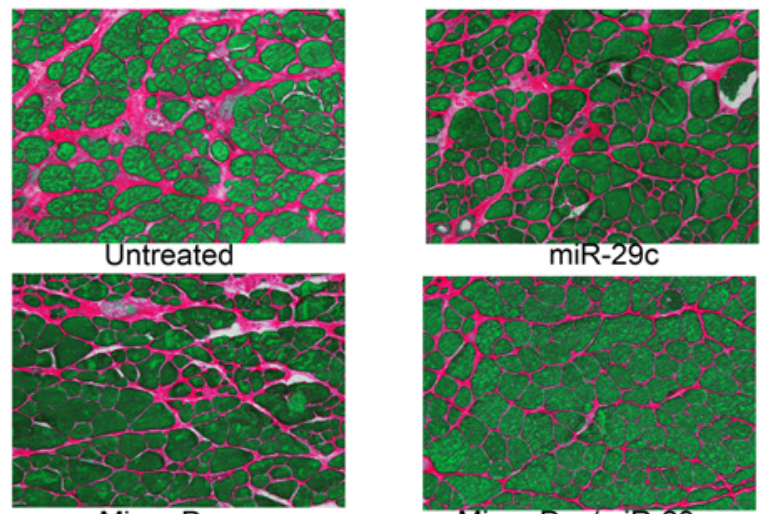

miR-29c

Micro-Dys

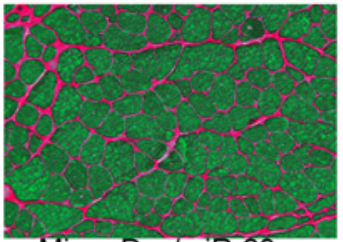

Micro-Dys/miR-29c

B
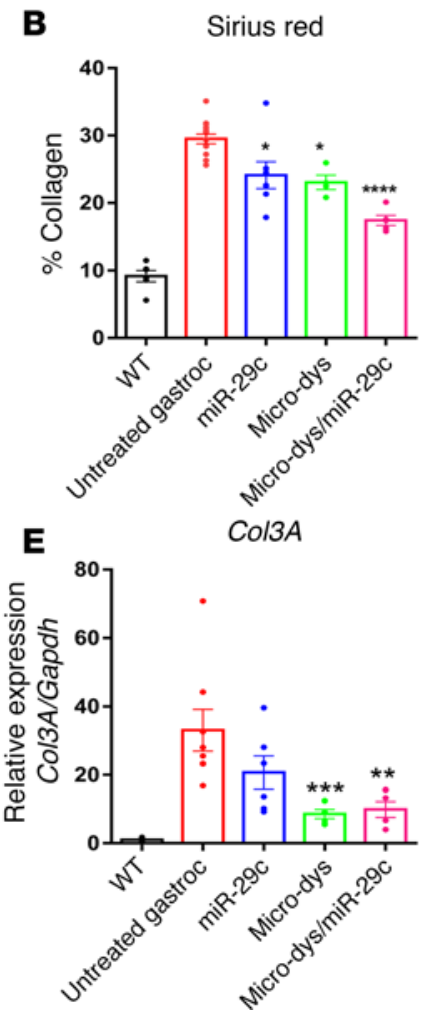

C
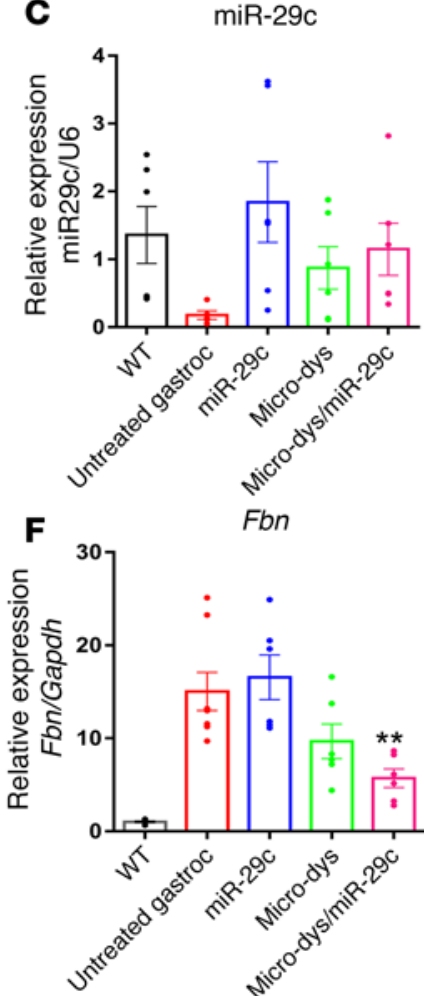

D
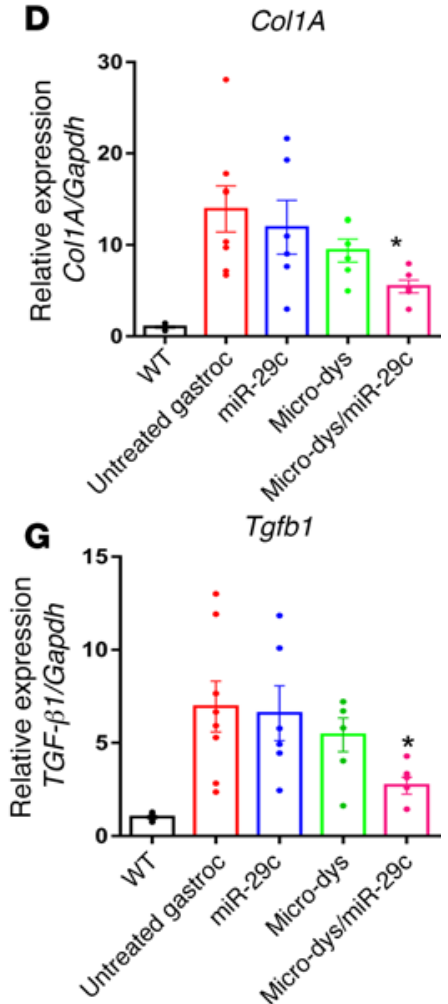

Figure 4. AAV.miR-29c/micro-dystrophin combination therapy is more effective at reducing fibrosis and extracellular matrix component expression. (A) Picrosirius red staining of untreated, AAV.miR-29c-, AAV.micro-dystrophin-, and AAV.miR-29c/AAV.micro-dystrophin-treated gastrocnemius (CAS) muscle 12 weeks after injection. Original magnification, $\times 20$. (B) Quantification of picrosirius red staining shows cotreated muscle had a $40.8 \%$ reduction in collagen compared with untreated GAS muscle ( $n=4-7$ per group except $n=14$ for untreated); 1 -way ANOVA. (C) qRT-PCR confirms an increase in miR-29c transcript levels in the treated cohorts. Semiquantitative qRT-PCR shows a significant reduction in Col1A and Col3A (D and E), Fbn (F), and Tgfb1 (C) levels in the AAV.miR-29c/AAV.micro-dystrophin-treated muscle compared with the contralateral limb and each of the single therapies. (C-C) $n=6$ per group except $n=8$ untreated; 1-way ANOVA. All data represent mean \pm SEM. ${ }^{*} P<0.05,{ }^{* *} P<0.01,{ }^{* *} P<0.001,{ }^{* * *} P<0.0001$.

the treatment with micro-dystrophin gene replacement could further enhance muscle function. Twelve weeks after injection, the GAS was isolated for in vivo force measurements. Combinatorially treated $m d x / \mathrm{utrn}^{+/}$ GAS muscle showed significant improvement in absolute force when compared with untreated muscle (combinatorial treatment: $3,582.4 \pm 79.4 \mathrm{mN}$; untreated: $1,722 \pm 145.7 \mathrm{mN} ; P<0.0001$ ) and no difference from WT $(3,005 \pm 167.3 \mathrm{mN})$. Normalized specific force improved in combinatorial treatment compared with untreated GAS muscle $\left(244.2 \pm 6.6 \mathrm{mN} / \mathrm{mm}^{2}\right.$ vs. $m d x / \mathrm{utrn}^{+/-}$untreated, $151.6 \pm 14.5 \mathrm{mN} / \mathrm{mm}^{2} ; P<$ 0.0005 ) (Figure 5, A and B). The combinatorial therapy also protected against repeat eccentric contractions compared with untreated $m d x / \mathrm{utrn}^{+/-}$(combinatorial $0.66 \pm 0.04$ vs. $0.54 \pm 0.06(P \leq 0.0001)$. However, 

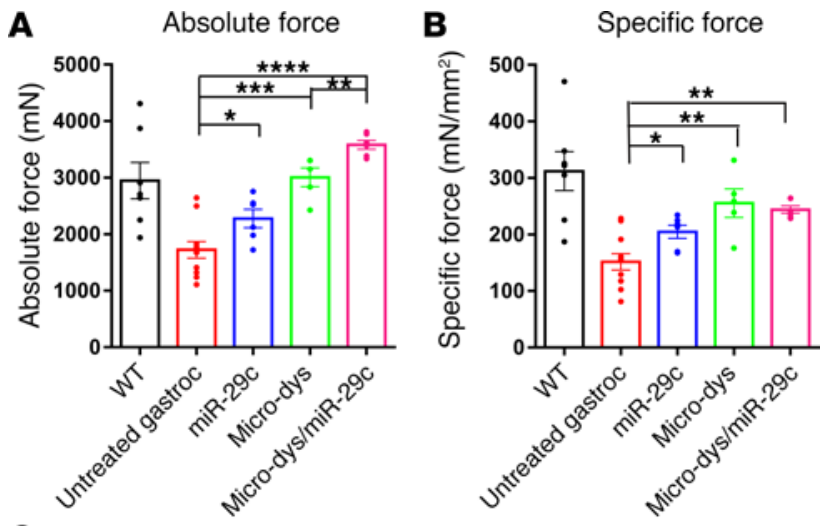

C

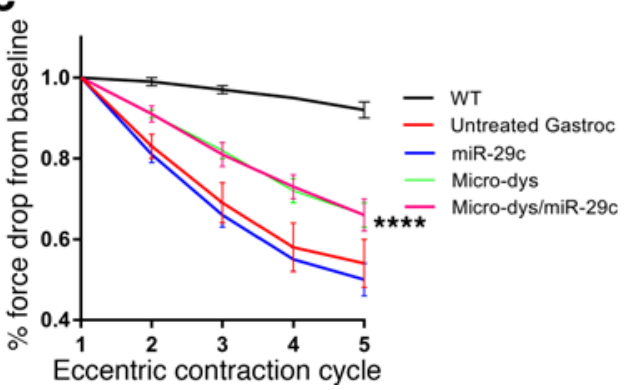

Figure 5. Combination therapy restores force and protects against contractioninduced damage. Absolute (A) and normalized specific force (B) following tetanic contraction in all 3 treatment-injected gastrocnemius muscles were significantly increased compared with untreated $m d x /$ utrn $^{+/-}$muscle. ${ }^{*} P<0.05$, ${ }^{* *} P$ $<0.01,{ }^{* * *} P<0.001,{ }^{* * *} P<0.0001 . n=5-7$ per group except $n=11$ untreated; 1 -way ANOVA. (C) Muscles were then assessed for loss of force following repetitive eccentric contractions. Only mice cotreated with miR-29c/micro-dystrophin and micro-dystrophin alone showed a protection from loss of force compared with untreated $m d x /$ utrn $^{+/-}$muscles (red). $n=5-6$ per group except $n=9$ untreated; 2-way ANOVA. ${ }^{* * *} P<0.0001$. All data represent mean \pm SEM.

there was no difference between micro-dystrophin alone compared with combinatorial therapy and neither achieved the protection seen in WT (decayed to $0.92 \pm 0.02$ ) (Figure $5 \mathrm{C}$ ). Similar findings were seen at 24 weeks after injection (Supplemental Figure 4, A-C).

Combination treatment increases muscle hypertrophy. We found that miR29c codelivered with micro-dystrophin increased the overall weight of the injected GAS compared with either one alone at 3 months (Supplemental Figure 3A) or 6 months after injection (Supplemental Figure 4D). To investigate the source of increased muscle mass, myofiber diameters were first measured. miR-29c/micro-dystrophin combination treatment resulted in an increase in average fiber size. Comparing $m d x / \mathrm{utrn}^{+/-}$controls with miR-29c/micro-dystrophin-treated $m d x / \mathrm{utrn}^{+/}$GAS, the average diameter increased from 25.96 to $30.97 \mu \mathrm{m}$ (Supplemental Figure 3B) at

3 months and from 27.9 to $33.2 \mu \mathrm{m}$ at 6 months after treatment (Supplemental Figure 4E). The codelivery produced a shift towards WT fiber size distribution (Supplemental Figure 3C and Supplemental Figure 4F). GAS muscles from all groups were full-slide scanned and the total area was measured. Muscles cotreated with micro-dystrophin/miR-29c had a significant increase in cross sectional area compared with untreated and either treatment alone (uninjected: $24.6 \mathrm{~mm}^{2}$ vs. miR-29c: $26.3 \mathrm{~mm}^{2}$ vs. micro-dystrophin: $26.6 \mathrm{~mm}^{2}$ vs. micro-dystrophin/miR-29c: $33.1 \mathrm{~mm}^{2}$ ) (Figure 6 and Supplemental Figure 3D). miR-29c has been reported to play a role in the myoD/Pax7/myogenin pathway and thus we hypothesized that it may be impacting regeneration and radial growth. To verify that the increase in gross muscle weight was not caused by hyperplasia, we counted the total number of muscle fibers from the full-slide-scanned images. We found that there was no difference in the total number of muscle fibers following miR-29c/micro-dystrophin combination treatment (Supplemental Figure 3E) compared with the untreated limb. Finally, given that muscle fiber diameters in $m d x / u^{+/-}$mice are heterogeneous, with many small fibers and some hypertrophic fibers, we asked whether the number of fibers per unit area (cells $/ \mathrm{mm}^{2}$ ) was affected with treatment. We found that miR-29c/microdystrophin combination treatment was not different than WT, with more uniform fibers compared with the untreated side, which contained more tiny fibers (Figure 4A and Supplemental Figure 3F). Interestingly, we only saw a difference in the number of fibers per unit area $\left(\right.$ cells $\left./ \mathrm{mm}^{2}\right)$ between untreated and WT muscle at age 6 months (Supplemental Figure 3F) and not at 9 months (Supplemental Figure 4G). These data support that the increase in weight following miR-29c gene transfer helps muscle fibers grow radially and convert a muscle environment previously supporting fibrogenesis into a more myogenic milieu.

Early treatment with AAV.miR-29c/micro-dystrophin combination prevents fibrosis. In view of the potential importance of combinatorial $\mathrm{miR}-29 \mathrm{c}$ and micro-dystrophin as a prophylactic therapy for DMD we also examined a cohort of younger $m d x /$ utrn $^{+/-}$mice, treated at 4 weeks of age. Using the same paradigm as for other groups, we compared efficacy for prevention of fibrosis by i.m. injection of GAS using scAAVrh.74. CMV.miR-29c alone, ssAAVrh74.MCK.micro-dystrophin + scAAVrh.74.CMV.miR-29c combination therapy, or ssAAVrh74.MCK.micro-dystrophin alone at the same dose. The mice were necropsied 12 weeks after injection. A significant decrease in collagen staining throughout the GAS muscles in all treated groups compared with the untreated contralateral $m d x /$ utrn $^{+/-}$GAS muscle was observed (Figure 7A). Quantification of the picrosirius red staining showed that muscle cotreated with micro-dystrophin/miR-29c had a $51 \%$ reduction in collagen compared with the untreated muscle (treated: $11.32 \% \pm 1.18 \%$ vs. untreated: $23.15 \% \pm$ $0.90 \%)(P<0.0001)$ (Figure 7B), and qRT-PCR confirmed Col1A, Col3A, Fbn, and Tgfb1 reduction following combinatorial therapy (Figure 7, D and $\mathrm{E}$ ). 


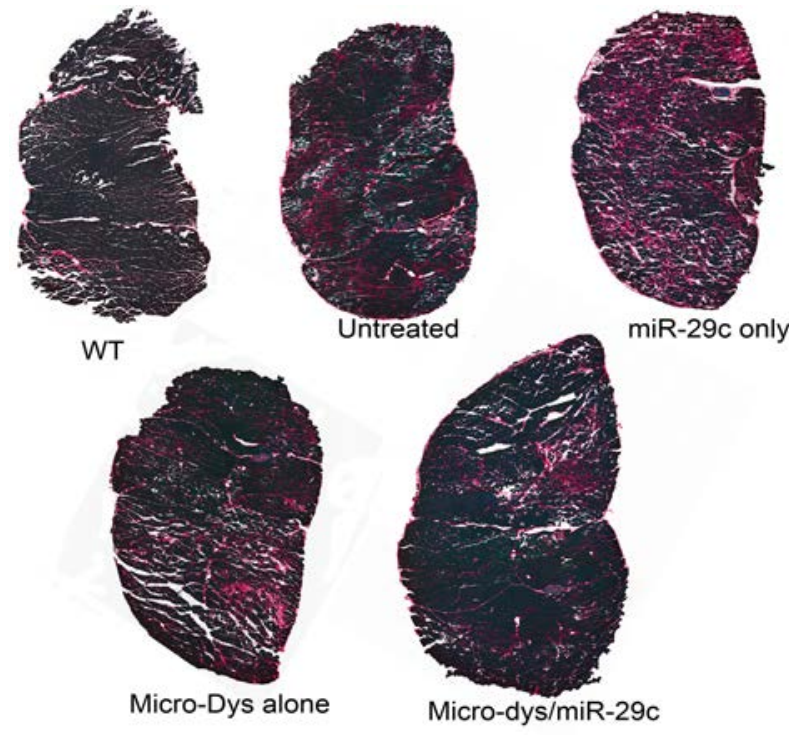

Micro-Dys alone
Figure 6. miR-29c/micro-dystrophin combination increases muscle size. Treated and untreated $m d x / u_{t r n}{ }^{+/}$gastrocnemius muscles were sectioned and stained with picrosirius red to detect collagen. Fibrotic areas are pink and intact muscle is green. On the macroscopic level, miR-29c/micro-dystrophin combination decreases fibrosis and increases total cross sectional area. Original magnification, $\times 2.5$.

Early combination therapy restores force and protects from contractioninduced damage better than late treatment. We again performed in vivo force measurement on the GAS. In 4-week-old $m d x /$ utrn $^{+/-}$mice, cotreatment using $\mathrm{miR}-29 \mathrm{c} / \mathrm{micro}-d y s t r o p h i n$ showed significant improvement in absolute force when compared with untreated $m d x / \mathrm{utrn}^{+/-}$mice and there was no difference from WT (cotreated: $2,908 \pm 129.5 \mathrm{mN}$ vs. untreated: $1,639.4 \pm 116.9 \mathrm{mN}$ vs. WT: $3,369.73 \pm 154.1 \mathrm{mN}$ ). Specific force was also normalized to WT levels following combinatorial therapy (cotreated: $338.9 \pm 22.34 \mathrm{mN} / \mathrm{mm}^{2}$ vs. untreated: $184.3 \pm 13.42 \mathrm{mN} / \mathrm{mm}^{2}$ vs. WT: $364.3 \pm 7.79 \mathrm{mN} / \mathrm{mm}^{2}$ ) (Figure 8, A and B).

Next, each GAS was subjected to a series of repeat eccentric contractions. By comparing the force ratio of each contraction by the fifth contraction, untreated muscle decayed to $0.53 \pm 0.04$ versus cotreated 0.82 \pm 0.04 ( $P \leq 0.0001)$. The combinatorial treatment group was slightly lower than WT, which decayed to $0.93 \pm 0.01$, but not significantly different (Figure $8 \mathrm{C}$ ). These data show that reducing fibrosis and gene replacement leads to increases in both absolute and specific force and significantly protects muscle from contraction-induced injury.

\section{Discussion}

One of the potential pitfalls to achieving success using current gene replacement strategies for DMD is the presence of fibrosis that may hinder the full potential of gene replacement $(30,31)$. In the current study, we provide evidence for the use of AAV-mediated overexpression of miR-29c, codelivered with micro-dystrophin, as a potential therapeutic approach for the treatment of DMD. We compared multiple paradigms to suppress skeletal muscle fibrosis in an animal model suitable to test and compare modes of treatment. The animal model used for proof of principle was the $m d x / \mathrm{utrn}^{+/-}$mouse, known for the extent of fibrosis found in the GAS muscle as early as 3 months of age (19). We constructed a potentially promising vector for translational studies using AAV containing miR-29c expressed from a miR-30 backbone in an EF1 $\alpha$ intron driven by a CMV promoter. A proof-of-principle in vitro study of this cassette transfected with a luciferase expression plasmid containing the COL1A13'UTR, which harbors multiple miR-29 binding sites, resulted in significant repression of luciferase activity; comparable studies using a mutant COL1A1 3'UTR had no effect on luciferase. Successful studies in cell culture were followed by i.m. delivery to the GAS of $m d x /$ utrn $^{+/-}$mice using scAAVrh.74.CMV.miR-29c at a dose safe for translation, $5 \times 10^{11} \mathrm{vg}$. This resulted in a significant decrease in collagen throughout the GAS muscle (Figure 2). We then turned to combinatorial treatment using codelivery of miR-29c and micro-dystrophin injected into GAS. The picrosirius-stained, postinjected muscle demonstrated a $41 \%$ reduction in collagen at 3 months and $47 \%$ decrease at 6 months compared with untreated muscle (Figure 4). Muscle fiber size also showed a modest increase at 3 months after gene transfer with a shift toward WT fiber size distribution (Supplemental Figures 3 and 4 ) that was sustained in the 6-month posttreatment muscle analysis. Combinatorial delivery also showed reduced levels of TGF- $\beta 1$, providing insight and a possible explanation for the reduction of collagen (Figure 4).

The benefits of combinatorial therapy extended to functional efficacy, helping to provide a stronger rational for clinical translation. Although miR-29c overexpression alone increased absolute and specific force (Figure 3), it did not protect against eccentric contraction-induced injury. Eccentric contractions are a frequent cause of muscle injury and considering the membrane fragility associated with dystrophin deficiency, correcting this functional defect could have important clinical implications. Based on this premise, we tested combinatorial therapy replacing micro-dystrophin along with overexpression of miR-29c and found protection against repeated eccentric contractions $(P \leq 0.0001)$. There was, however, no difference between micro-dystrophin alone compared with combinatorial therapy and neither achieved the level of protection seen in WT (Figure 5). This may in fact indicate that the level of existing 
A

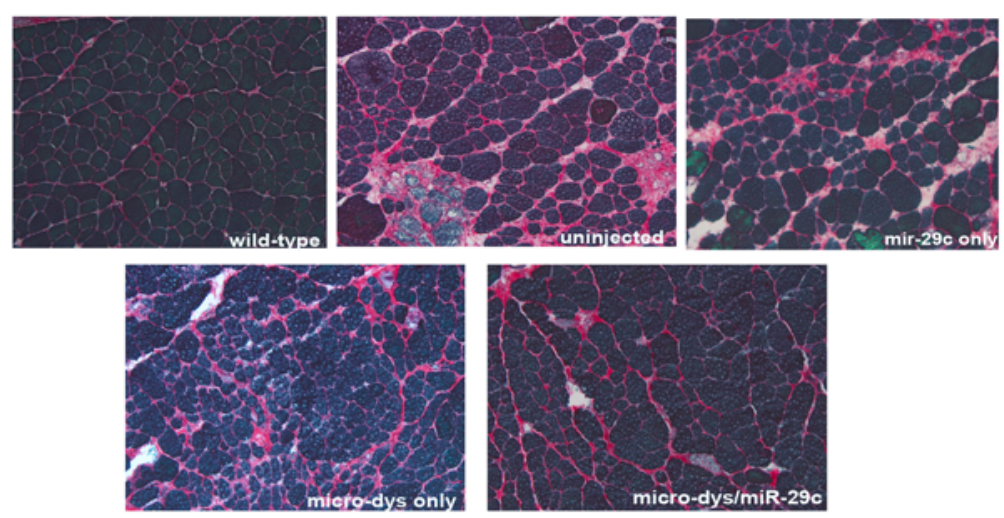

B
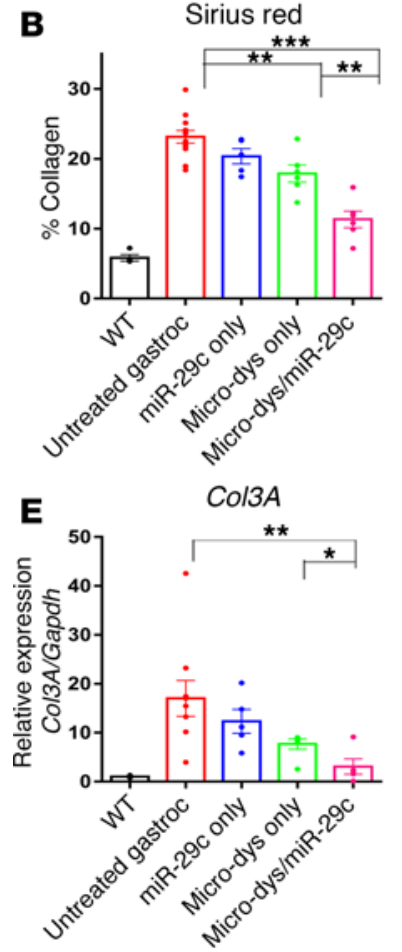

C
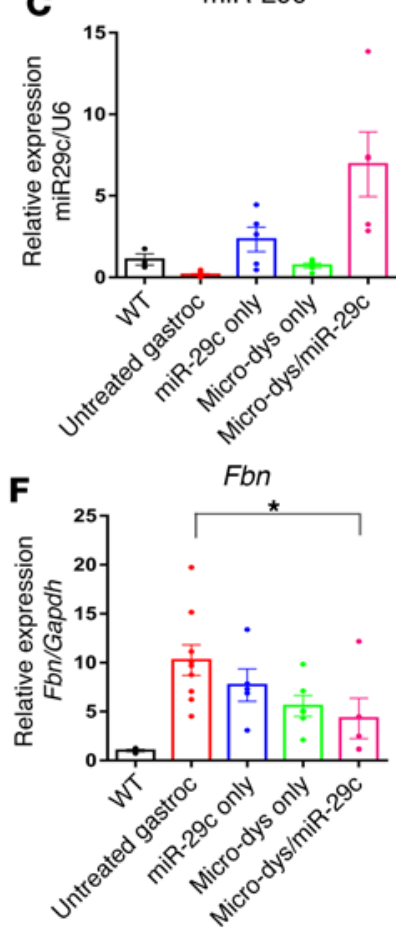
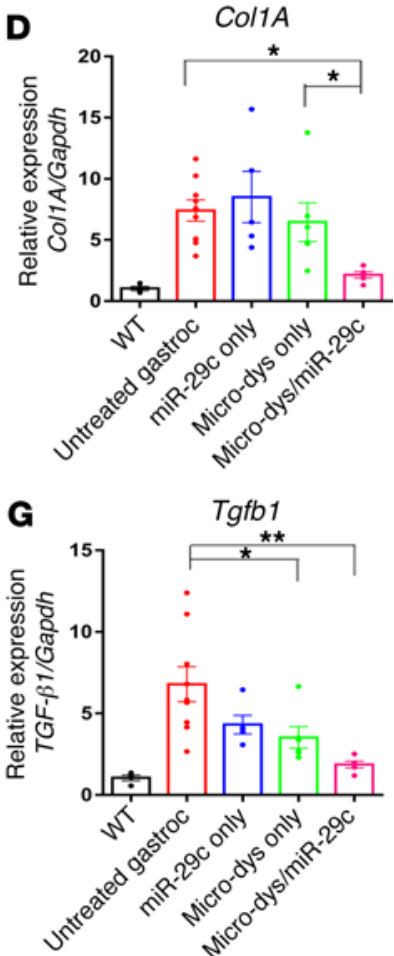

Figure 7. Early treatment with AAV.miR-29c/micro-dystrophin combination therapy is more effective at reducing fibrosis and extracellular matrix component expression. (A) Picrosirius red staining of gastrocnemius (GAS) muscle from WT, untreated, AAV.miR-29c-, AAV.micro-dystrophin-, and AAV.miR29c/AAV.micro-dystrophin-treated mice injected at 4 to 5 weeks of age taken out 12 weeks after injection. Original magnification, $\times 20$. (B) Quantification of picrosirius red staining shows cotreated muscle had a 51.1\% reduction in collagen compared with untreated GAS muscle ( $n=4-6$ per group except $n=12$ untreated); 1-way ANOVA. (C) qRT-PCR confirms an increase in miR-29c transcript levels in the treated cohorts ( $n=3-5$ for all groups except $n=7$ untreated); 1-way ANOVA. Semiquantitative qRT-PCR shows a significant reduction in Col1A1 and Col3A1 (D and E), Fbn (F), and Tgfb1 (G) levels in the AAV.miR-29c/AAV. micro-dystrophin-treated muscle compared with the contralateral limb and each of the single therapies. (D-G) $(n=4-6$ per group except $n=9$ untreated); 1-way ANOVA. All data represent mean \pm SEM. ${ }^{*} P<0.05,{ }^{* *} P<0.01,{ }^{* * *} P<0.001$.

fibrosis present will be the factor that determines the extent of treatment. Extended time points will help elucidate the long-term benefit of mir-29C overexpression. When treatment was administered to young $m d x / u_{t r n}^{+/-}$mice there was efficacy beyond that seen in older mice. Gene transfer of combinatorial miR-29c and micro-dystrophin at 4 weeks had a greater impact on every parameter measured. Fibrosis was reduced by $51 \%$ versus $40 \%$ following treatment delayed to 3 months of age (Figure 7 ). We also studied specific force, absolute force, and protection against eccentric contraction in the cohort of younger $m d x /$ utrn $^{+/-}$mice; all of these outcome measures were equivalent to WT results following gene transfer (Figure 8). This bodes well for enhancing translational gene replacement when it is started in the newborn period. Efforts are clearly moving in the direction of identifying DMD and other muscular dystrophies in the newborn period. The Ohio Newborn Screening Study (32) illustrates the potential for 

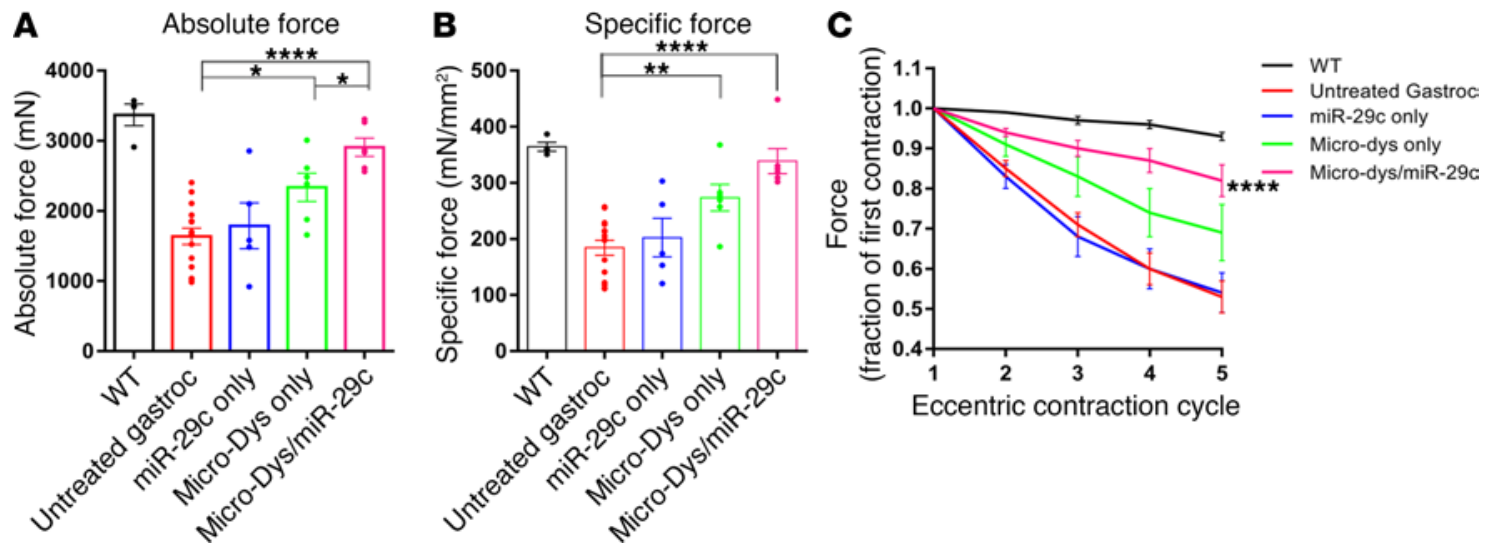

Figure 8. Early combination therapy restores force and protects against contraction-induced damage. Absolute (A) and normalized specific force (B) following tetanic contraction in all 3 treatment-injected gastrocnemius muscles were significantly increased compared with untreated $m d x / \mathrm{utrn}^{+/}$ muscle. (A and B) $n=4-6$ per group except $n=15$ for untreated); 1-way ANOVA. (C) Muscles were then assessed for loss of force following repetitive eccentric contractions. Only mice cotreated with miR-29c/micro-dystrophin and micro-dystrophin alone showed a protection from loss of force compared with untreated $m d x /$ utrn $^{+/-}$muscles (red). $n=4-6$ for per group except $n=15$ untreated; 2-way ANOVA. All data represent mean \pm SEM. ${ }^{*} P$ $<0.05,{ }^{* *} P<0.01,{ }^{* * *} P<0.0001$.

identification of DMD in newborns using creatine kinase as a biomarker (>2,000 U/1) with DNA confirmation on the same dried blood spot. This methodology is now being extended to other states in the USA (Parent Project Muscular Dystrophy [PPMD] May 16, 2016: Next Steps with Newborn Screening; http://community.parentprojectmd.org/profiles/blogs/next-steps-with-newborn-screening) and in other countries, particularly China (Perkin Elmer launches screening in China; http://www.genengnews. com/gen-news-highlights/perkinelmer-launches-newborn-screening-program-in-china/81249941).

miR-29 has also shown promise as a treatment modality for cardiac, pulmonary, and liver fibrosis. Myocardial infarction in mice and humans is associated with miR-29 downregulation. Rooij et al. (7) demonstrated that exposing fibroblasts to a miR-29b mimic decreased collagen transcripts, providing a path for clinical translation for cardiac fibrosis. Subsequent studies showed that in a bleomycin-induced pulmonary fibrosis mouse model, attenuation of fibrosis could be achieved using the Sleeping Beauty (SB) transposon system-based delivery of miR-29b (15). Currently, a miR-29b mimic is in a clinical phase 1 safety-tolerability local intradermal trial in healthy volunteers (miRagen Therapeutics MRG-201). These studies support the rationale for testing AAV gene delivery of miR-29 in muscular dystrophy with the advantage of more complete correction when potentiated by concurrent correction of dystrophin deficiency. Collectively, these studies provide a path for a translational safety and efficacy clinical trial in DMD patients. Compared with miR-29 oligonucleotide delivery that would require repeated administration related to the half-life of the oligonucleotides, AAV gene therapy could potentially provide a path for single-delivery gene transfer.

\section{Methods}

Mice

C57BL/10 mice were purchased from the Jackson Laboratory. $m d x /$ utrn ${ }^{+/-}$mice were generated by breeding $m d x /$ utrn $^{+/-}$mice, a gift from Jill Rafael-Fortney (The Ohio State University). Identification of $m d x /$ utrn $^{+/-}$mice was done by genotyping. All animals were housed in standard mouse cages with food and water ad libitum. Both male and female mice were used in these studies. Mice were injected at 12 weeks of age for the late treatment study and at 4 to 5 weeks of age for the early treatment study.

\section{Genotyping}

$m d x /$ utrn $^{+/-}$mice were identified by genotyping. DNA from tail clippings was analyzed by using OneTaq DNA Polymerase (New England Biolabs) PCR. PCR analysis to determine utrophin-knockout status used a forward primer complementary to exon 7 of mouse utrophin (5'-GTGAAGGATGTCATGAAAG-3') and reverse primers complementary to either intron 7 (5'-TGAAGTCCGAAAGAGATACC-3') or to the PGK promoter located within the Neo-knockout cassette (5'-ACGAGACTAGTGAGACGTGC-3'). 
Reactions were carried out on genomic DNA for 30 cycles under the following conditions: $94^{\circ} \mathrm{C}, 30 \mathrm{sec}-$ onds; $57^{\circ} \mathrm{C}, 30$ seconds; $68^{\circ} \mathrm{C}, 20$ seconds.

\section{DNA constructs and AAV production}

The miR-29c cDNA was synthesized by ordering a custom DNA sequence from GenScript containing miR-29c embedded in a miR-30 backbone. Three bases of the miR-29c sequence were modified. This sequence was then cloned into an SCAAV ITR-containing plasmid driven by the CMV promoter and polyA sequence. A modified cross-packaging approach, which we have previously reported (4), was used to produce the rAAV vector. Here, a triple-transfection method with $\mathrm{CaPO}_{4}$ precipitation in HEK293 cells allows for AAV2 ITRs to be packaged into a different AAV capsid serotype (33, 34). The production plasmids were: (a) pAAV.CMV.miR-29c or pAAV.MCK.micro-dystrophin (4), (b) rep2-caprh.74modified AAV helper plasmids encoding cap serotype 8-like isolate rh.74, and (c) an adenovirus type 5 helper plasmid (pAdhelper) expressing adenovirus E2A, E4 ORF6, and VA I/II RNA genes. Vectors were purified and encapsidated vg titer (utilizing a Prism 7500 Taqman detector system (PE Applied Biosystems) was determined as previously described (35). The primer and fluorescent probe targeting the MCK promoter were: MCK forward primer, 5'-CCCGAGATGCCTGGTTATAATT-3'; MCK reverse primer, 5'-GCTCAGGCAGCAGGTGTTG-3'; and MCK probe, 5'-FAM-CCAGACATGTGGCTGCTCCCCC -TAMRA-3'. The primer and fluorescent probe targeting the CMV promoter were: CMV forward primer, 5'-TGGAAATCCCCGTGAGTCAA-3'; CMV reverse primer, 5'-CATGGTGATGCGGTTTTGG-3'; and CMV probe, 5'-FAM-CCGCTATCCACGCCCATTGATG-TAMRA-3'.

\section{Luciferase assay}

The WT and mutant COL1A1 3'UTR constructs were previously described (14). COL1A1 reporter plasmid (100 ng) and $50 \mathrm{ng}$ of Renilla luciferase control plasmid (phRL-SV40, from Promega) with varying concentrations of miR-29c plasmid were transfected using Lipofectamine 2000 (Invitrogen) according to the manufacturer's instructions into HEK293 cells. Luciferase assays were performed 20 hours after transfection using the Dual Luciferase Reporter Assay System (Promega). Each transfected well was assayed in triplicate. Firefly luciferase activity was normalized to Renilla luciferase activity for each transfected well.

\section{Animal studies}

Gene delivery - i.m. injection. Mice were anesthetized and maintained under $1 \%-3 \%$ isoflurane (in $\mathrm{O}_{2}$ ). The posterior compartment of the lower left limb of 4 - and 12 -week-old $m d x / \mathrm{utrn}^{+/-}$mice was cleaned with $95 \%$ ethanol and then the GAS was injected with $5 \times 10^{11} \mathrm{vg}$ of scAAVrh.74.CMV.miR-29c, $5 \times 10^{11} \mathrm{vg}$ of scAAVrh.74.MCK.micro-dystrophin, or both diluted in saline in 50 to $80 \mu 1$ using a 30-gauge ultrafine insulin syringe. Mice receiving combinatorial therapy received a total virus dose of $1 \times 10^{12} \mathrm{vg}$. The contralateral muscle was left untreated to serve as a control. GAS muscle from both limbs was removed at 12 and 24 weeks after injection to assess gene transfer efficiency and physiology.

Force generation and protection from eccentric contractions. The GAS procedure follows the protocol listed in Hakim et al. (36) for TA muscle physiology but adapted for the GAS. Mice were anesthetized using a ketamine/xylazine mixture. The hind limb skin was removed to expose the GAS muscle and the Achilles tendon. The distal tendon was dissected out and a double-square knot was tied around the tendon with 4-0 suture as close to the muscle as possible; another double-square knot was tied right next to the first knot and then the tendon was cut. The exposed muscle was constantly dampened with saline. Mice were then transferred to a thermally controlled platform and maintained at $37^{\circ} \mathrm{C}$. The knee was secured to the platform with a needle through the patella tendon, and the tendon sutured to the level arm of a force transducer (Aurora Scientific), and the foot was secured with tape. The GAS muscle contractions were elicited by stimulating the sciatic nerve via bipolar platinum electrodes. Once the muscle was stabilized, the optimal length was determined by incrementally stretching the muscle until the maximum twitch force was achieved. After a 3-minute rest period, the GAS was stimulated at $50,100,150$, and $200 \mathrm{~Hz}$, allowing a 1-minute rest period between each stimulus to determine maximum tetanic force. Muscle length was measured. Following a 5-minute rest, the susceptibility of the GAS muscle to contraction-induced damage was assessed. After $500 \mathrm{~ms}$ of stimulation, the muscle was lengthened by $10 \%$ of the optimal length. This consisted of stimulating the muscle at $150 \mathrm{~Hz}$ for 700 ms. After the stimulation, the muscle was returned to the optimal length. The cycle was repeated every 
minute for a total of 5 cycles. Specific force was calculated by dividing the maximum tetanic force by the GAS muscle cross sectional area. After the eccentric contractions, the mice were then euthanized and the GAS muscle was dissected out, weighed, and frozen for analysis.

\section{Picrosirius red stain and collagen quantification}

Frozen sections placed onto Fisherbrand Superfrost charged microscope slides were fixed in 10\% neutral buffered formalin for 5 minutes, and then rinsed in distilled water. Slides were then incubated in Solution B (Direct Red 80/2,4,6-trinitrophenol) from the Picrosirius Red Stain Kit (Polysciences Inc., catalog 24901) for 15 minutes. After a thorough rinse in distilled water, the slides were placed in Solution C $(0.1 \mathrm{~N}$ hydrochloride acid) for 2 minutes. Slides were counterstained for 3 minutes with $1 \%$ Fast Green in 1\% glacial acetic acid from Poly Scientific (catalog S2114) using a 1:10 dilution in deionized water. Finally, the slides were rinsed again in distilled water, dehydrated in graded ethanol, cleared in xylene, and mounted with coverslips using Cytoseal 60 media from Thermo Fisher Scientific (catalog 8310). Images were taken using the AxioVision 4.9.1 software. For analysis of picosirius red staining and percentage collagen quantification, the contrast between the red and the green colors were enhanced using Adobe Photoshop. The color deconvolution plugin in the ImageJ software program (NIH) was selected and the RGB color deconvolution option was used. The red image includes all connective tissue from the picosirius red stain. The green image includes all muscle from the Fast Green counterstain. Only the red image and the original image were used. A threshold was then applied to the images to obtain black and white images with areas positive for collagen in black and negative areas in white. Using the measure function, the area of collagen was calculated. The total tissue area was then determined by converting the original image to 8-bit and adjusting the threshold to 254, which is 1 unit below complete image saturation. The total tissue area was then measured as described above and the total area was recorded. The percentage of collagen was calculated by dividing the area of collagen by the total tissue area. The mean percentage for each individual was then calculated.

\section{Gene expression analysis}

GAS muscles were embedded in 7\% gum tragacanth and flash frozen in isopentane cooled in liquid nitrogen. Cryostat sections $(12 \mu \mathrm{m})$ for immunostains were incubated with anti-flag primary antibody (Sigma-Aldrich, catalog F7425) at a 1:175 dilution in blocking buffer ( $1 \times$ TBS, $10 \%$ goat serum, $0.1 \%$ Tween) for 1 hour at room temperature in a wet chamber. Sections were then washed in TBS 3 times for 20 minutes each and then reblocked. Slides were incubated for 45 minutes at room temperature with Alexa Fluor 568 goat anti-rabbit IgG at a 1:300 dilution (Molecular Probes, catalog A-11011). Sections were washed in TBS 3 times for 20 minutes each and then mounted with Vectashield mounting medium (Vector Laboratories). The number of fibers with sarcolemmal dystrophin staining is expressed as the percentage of dystrophin-positive fibers.

\section{qRT-PCR}

Total RNA (including miR) from muscle was extracted using TRIzol (Invitrogen). Expression of mature miR was determined by the miR-specific Taqman MicroRNA Assay Kit (Life Technologies) using the ABI PRISM 7500 Detection System. U6 was used for normalization. Analysis of mRNA expression was also done for Col1A1, Col3A1, Fbn, and Tgfb using gene-specific TaqMan primers/probes (Life Technologies). GAPDH was used for normalization.

\section{Histology and morphometric analysis}

GAS muscles from 16-, 24-, and 36-week-old mice, treated with scAAVrh.74.CMV.miR-29c, ssAAVrh.74. MCK.micro-dystrophin, or both versus untreated, were stained with hematoxylin and eosin, cut in cross section, and photographed using Zeiss Axiovision L4 software ( 6 random $\times 20$ images per section per animal). Fiber diameters were compared between all treated and all control muscles. Approximately 700 1,200 fibers were counted per muscle per animal. Full-slide scans were done by the Nationwide Children's Hospital (NCH) Biopathology Center. Total area was measured using ImageScope Software.

\section{Statistics}

All data are expressed as the mean \pm SEM unless otherwise indicated. Statistical differences between 2 experiment groups were determined using unpaired 2-tailed Student's $t$ test. Statistical differences between multiple experimental groups were determined using a 1-way or 2-way ANOVA. All statistical 
analyses were performed using GraphPad Prism 7.0. A $P$ value less than 0.05 was considered statistically significant.

\section{Study approval}

All animal protocols and procedures were reviewed and approved by The Research Institute at NCH Institutional Animal Care and Use Committee, Columbus, OH (protocol AR06-00054).

\section{Author contributions}

$\mathrm{KNH}$, JRM, and LRK conceived of the study and designed the experiments. KNH performed all of the experiments. JTM provided the miR-30 backbone, assisted with construct design, and provided guidance for luciferase assays. KNH, JRM, and LRK wrote the manuscript. All authors reviewed, edited, and approved the manuscript.

\section{Acknowledgments}

We would like to thank the NCH Viral Vector Manufacturing Facility for supplying vectors for this study and the NCH Biopathology Center for full-slide scanning. We would also like to thank Eric Meadows for cloning the pAAV.CMV.miR-29c and performing the luciferase assays. This work has been funded by Jesse's Journey (LRK) and the NIH (R35CA197311 to JTM). JTM is an Investigator of the Howard Hughes Medical Institute.

Address correspondence to: Louise R. Rodino-Klapac, Center for Gene Therapy, The Research Institute at Nationwide Children's Hospital, Room 3016, 700 Childrens Drive, Columbus, Ohio 43205, USA. Phone: 614.355.2694; E-mail: louise.rodino-klapac@nationwidechildrens.org.

1. Rodino-Klapac LR, et al. Persistent expression of FLAG-tagged micro dystrophin in nonhuman primates following intramuscular and vascular delivery. Mol Ther. 2010;18(1):109-117.

2. Liu M, Yue Y, Harper SQ, Grange RW, Chamberlain JS, Duan D. Adeno-associated virus-mediated microdystrophin expression protects young $\mathrm{mdx}$ muscle from contraction-induced injury. Mol Ther. 2005;11(2):245-256.

3. Harper SQ, et al. Modular flexibility of dystrophin: implications for gene therapy of Duchenne muscular dystrophy. Nat Med. 2002;8(3):253-261.

4. Rodino-Klapac LR, et al. A translational approach for limb vascular delivery of the micro-dystrophin gene without high volume or high pressure for treatment of Duchenne muscular dystrophy. J Transl Med. 2007;5:45.

5. Wang B, et al. Suppression of microRNA-29 expression by TGF- $\beta 1$ promotes collagen expression and renal fibrosis. J Am Soc Nephrol. 2012;23(2):252-265.

6. Roderburg C, et al. Micro-RNA profiling reveals a role for miR-29 in human and murine liver fibrosis. Hepatology. 2011;53(1):209-218

7. van Rooij E, et al. Dysregulation of microRNAs after myocardial infarction reveals a role of miR-29 in cardiac fibrosis. Proc Natl Acad Sci USA. 2008;105(35):13027-13032.

8. Maurer B, et al. MicroRNA-29, a key regulator of collagen expression in systemic sclerosis. Arthritis Rheum. 2010;62(6):1733-1743.

9. Wang L, et al. Loss of miR-29 in myoblasts contributes to dystrophic muscle pathogenesis. Mol Ther. 2012;20(6):1222-1233.

10. Cushing L, et al. miR-29 is a major regulator of genes associated with pulmonary fibrosis. Am J Respir Cell Mol Biol. 2011;45(2):287-294

11. Eisenberg I, et al. Distinctive patterns of microRNA expression in primary muscular disorders. Proc Natl Acad Sci USA. 2007;104(43):17016-17021.

12. He Y, Huang C, Lin X, Li J. MicroRNA-29 family, a crucial therapeutic target for fibrosis diseases. Biochimie. 2013;95(7):1355-1359.

13. Dai Y, Dai D, Mehta JL. MicroRNA-29, a mysterious regulator in myocardial fibrosis and circulating miR-29a as a biomarker. J Am Coll Cardiol. 2014;64(20):2181.

14. Knabel MK, et al. Systemic delivery of scAAV8-encoded MiR-29a ameliorates hepatic fibrosis in carbon tetrachloride-treated mice. PLoS One. 2015;10(4):e0124411.

15. Xiao J, et al. miR-29 inhibits bleomycin-induced pulmonary fibrosis in mice. Mol Ther. 2012;20(6):1251-1260.

16. Wang H, et al. NF-kappaB-YY1-miR-29 regulatory circuitry in skeletal myogenesis and rhabdomyosarcoma. Cancer Cell. 2008;14(5):369-381.

17. Wei W, et al. miR-29 targets Akt3 to reduce proliferation and facilitate differentiation of myoblasts in skeletal muscle development. Cell Death Dis. 2013;4:e668.

18. McDonald AA, Hebert SL, Kunz MD, Ralles SJ, McLoon LK. Disease course in mdx:utrophin ${ }^{+/}$mice: comparison of three mouse models of Duchenne muscular dystrophy. Physiol Rep. 2015;3(4):e12391.

19. Gutpell KM, Hrinivich WT, Hoffman LM. Skeletal muscle fibrosis in the $\mathrm{mdx} / \mathrm{utrn}^{+/}$mouse validates its suitability as a murine model of Duchenne muscular dystrophy. PLoS One. 2015;10(1):e0117306.

20. Zhou L, et al. Haploinsufficiency of utrophin gene worsens skeletal muscle inflammation and fibrosis in mdx mice. J Neurol Sci. 2008;264(1-2):106-111. 
21. Kota J, et al. Therapeutic microRNA delivery suppresses tumorigenesis in a murine liver cancer model. Cell. 2009;137(6):1005-1017.

22. Karakikes I, et al. Therapeutic cardiac-targeted delivery of miR-1 reverses pressure overload-induced cardiac hypertrophy and attenuates pathological remodeling. J Am Heart Assoc. 2013;2(2):e000078.

23. Dickins RA, et al. Probing tumor phenotypes using stable and regulated synthetic microRNA precursors. Nat Genet. 2005;37(11):1289-1295.

24. Wakabayashi-Ito N, Nagata S. Characterization of the regulatory elements in the promoter of the human elongation factor-1 alpha gene. J Biol Chem. 1994;269(47):29831-29837.

25. Willcocks RJ, et al. Multicenter prospective longitudinal study of magnetic resonance biomarkers in a large duchenne muscular dystrophy cohort. Ann Neurol. 2016;79(4):535-547.

26. Tandon A, et al. Dystrophin genotype-cardiac phenotype correlations in Duchenne and Becker muscular dystrophies using cardiac magnetic resonance imaging. Am J Cardiol. 2015;115(7):967-971.

27. Iannaccone $S$, et al. Connective tissue proliferation and growth factors in animal models of Duchenne muscular dystrophy. J Neurol Sci. 1995;128(1):36-44.

28. Bernasconi $\mathrm{P}$, et al. Expression of transforming growth factor-beta 1 in dystrophic patient muscles correlates with fibrosis. Pathogenetic role of a fibrogenic cytokine. J Clin Invest. 1995;96(2):1137-1144.

29. Yamazaki M, et al. Expression of transforming growth factor-beta 1 and its relation to endomysial fibrosis in progressive muscular dystrophy. Am J Pathol. 1994;144(2):221-226.

30. Yue Y, et al. A single intravenous injection of adeno-associated virus serotype-9 leads to whole body skeletal muscle transduction in dogs. Mol Ther. 2008;16(12):1944-1952.

31. Wang Z, et al. Successful regional delivery and long-term expression of a dystrophin gene in canine muscular dystrophy: a preclinical model for human therapies. Mol Ther. 2012;20(8):1501-1507.

32. Mendell JR, et al. Evidence-based path to newborn screening for Duchenne muscular dystrophy. Ann Neurol. 2012;71(3):304-313.

33. Rabinowitz JE, et al. Cross-packaging of a single adeno-associated virus (AAV) type 2 vector genome into multiple AAV serotypes enables transduction with broad specificity. J Virol. 2002;76(2):791-801.

34. Grieger JC, Choi VW, Samulski RJ. Production and characterization of adeno-associated viral vectors. Nat Protoc. 2006;1(3):1412-1428.

35. Clark KR, Liu X, McGrath JP, Johnson PR. Highly purified recombinant adeno-associated virus vectors are biologically active and free of detectable helper and wild-type viruses. Hum Gene Ther. 1999;10(6):1031-1039.

36. Hakim CH, Li D, Duan D. Monitoring murine skeletal muscle function for muscle gene therapy. Methods Mol Biol. 2011;709:75-89. 